\title{
Laparoscopic versus Open Surgery for Hepatocellular Carcinoma: A Meta-Analysis of High-Quality Case-Matched Studies
}

\author{
Ke Chen, ${ }^{1}$ Yu Pan, ${ }^{1}$ Bin Zhang, ${ }^{1}$ Xiao-long Liu, ${ }^{1}$ Hendi Maher, ${ }^{2}$ and Xue-yong Zheng ${ }^{1}$ \\ ${ }^{1}$ Department of General Surgery, Sir Run Run Shaw Hospital, School of Medicine, Zhejiang University, 3 East Qingchun Road, \\ Hangzhou, Zhejiang 310016, China \\ ${ }^{2}$ School of Medicine, Zhejiang University, 866 Yuhangtang Road, Hangzhou, Zhejiang 310058, China
}

Correspondence should be addressed to Xue-yong Zheng; 3306053@zju.edu.cn

Received 5 November 2017; Revised 5 December 2017; Accepted 23 January 2018; Published 1 March 2018

Academic Editor: Kevork M. Peltekian

Copyright (c) $2018 \mathrm{Ke}$ Chen et al. This is an open access article distributed under the Creative Commons Attribution License, which permits unrestricted use, distribution, and reproduction in any medium, provided the original work is properly cited.

\begin{abstract}
Objective. To present a meta-analysis of high-quality case-matched studies comparing laparoscopic (LH) and open hepatectomy $(\mathrm{OH})$ for hepatocellular carcinoma (HCC). Methods. Studies published up to September 2017 comparing LH and OH for HCC were identified. Selection of high-quality, nonrandomized comparative studies (NRCTs) with case-matched design was based on a validated tool (Methodological Index for Nonrandomized Studies) since no randomized controlled trials (RCTs) were published. Morbidity, mortality, operation time, blood loss, hospital stay, margin distance, recurrence, and survival outcomes were compared. Subgroup analyses were carried out according to the surgical extension (minor or major hepatectomy). Results. Twenty studies with a total of 830 patients ( 388 in $\mathrm{LH}$ and 442 in $\mathrm{OH}$ ) were identified. For short-term surgical outcomes, LH showed less morbidity (RR $=0.55 ; 95 \%$ CI, 0.47 0.65; $P<0.01)$, less mortality $(\mathrm{RR}=0.43 ; 95 \% \mathrm{CI}, 0.18 \sim 1.00 ; P=0.05)$, less blood loss $(\mathrm{WMD}=-93.21 \mathrm{ml}, 95 \%$ $\mathrm{CI},-157.33 \sim-29.09 \mathrm{ml} ; P<0.01$ ), shorter hospital stay (WMD $=-2.86,95 \% \mathrm{CI},-3.63 \sim-2.08 ; P<0.01$ ), and comparable operation time (WMD $=9.15 \mathrm{~min} ; 95 \% \mathrm{CI}:-7.61 \sim 25.90, P=0.28$ ). As to oncological outcomes, 5-year overall survival rate was slightly better in $\mathrm{LH}$ than $\mathrm{OH}(\mathrm{HR}=0.66,95 \% \mathrm{CI}$ : $0.52 \sim 0.84, P<0.01)$, whereas the 5 -year disease-free survival rate was comparable between two groups ( $\mathrm{HR}=0.88,95 \% \mathrm{CI}$ : $0.74 \sim 1.06, P=0.18$ ). Conclusion. This meta-analysis has highlighted that LH can be safely performed in selective patients and improves surgical outcomes as compared to $\mathrm{OH}$. Given the limitations of study design, especially the limited cases of major hepatectomy, methodologically high-quality comparative studies are needed for further evaluation.
\end{abstract}

\section{Introduction}

Although the incidence of hepatocellular carcinoma (HCC) has decreased, HCC is still the fifth most common malignancy and the third leading cause of cancer-related death worldwide [1]. Since laparoscopic hepatectomy (LH) was first reported in 1996 [2, 3], this treatment has been considered a landmark development in the progress of surgical treatment. However, the majority of HCC patients usually have cirrhosis and hypohepatia. Because of this, hepatectomy increases the risk of developing significant postoperative complications including ascites, hepatic failure, encephalopathy, and portal vein thrombosis [4]. There are some controversial aspects of LH for HCC including complications, postoperative recovery, and long-term survival outcomes.
During the last 6 years, a number of meta-analyses that compare LH with open hepatectomy $(\mathrm{OH})$ for HCC have been published [5-8]. Although randomized controlled trials (RCTs) are the most ideal tools for meta-analysis, no RCTs on this topic have been yet conducted. These meta-analyses included the available nonrandomized comparative studies (NRCTs) to overcome the paucity of RCTs. Therefore unreliable results and little strong evidence had been presented. On the other hand, there was evidence that estimates derived from high-quality NRCTs may be similar to those derived from RCTs [9]. Also, when comparing surgical procedures, pooling of high-quality NRCTs could be as accurate as pooling of RCTs [10]. In addition, several comparative studies on this topic have been published in the last 3 years and none of the published meta-analyses included studies published 
after 2013. Therefore, we performed an updated meta-analysis evaluating all of the available high-quality published trials to compare $\mathrm{LH}$ with $\mathrm{OH}$ for $\mathrm{HCC}$.

\section{Methods}

2.1. Systematic Literature Search. Systematic searches of PubMed, Embase, Cochrane Library, and Web of Science were performed to identify articles published up to September 2017. Searches included the terms "laparoscopic," "minimally invasive," "hepatectomy," "liver resection," "hepatocellular carcinoma," and "HCC". All eligible studies in English were retrieved, and their bibliographies were checked for potential relevant publications.

2.2. Eligibility Criteria and Quality Assessment. In order to reduce bias, our meta-analysis synthesized the existing observational studies while strictly limiting inclusion and exclusion criteria. First of all, papers containing any of the following were excluded: (1) studies that included malignant lesions other than HCC, (2) studies focusing on recurrent HCC, (3) studies that included cases of robotic-assisted hepatectomy. Secondly, only studies designed with casematched analysis were further evaluated and nonmatched studies were excluded. Then, the methodological quality of the eligible nonrandomized comparative studies (NRCTs) was assessed by the Methodological Index for Nonrandomized Studies (MINORS) [11]. In total, 8 items were evaluated, with a maximum score of 16 points. Studies with 12 or more points were considered of high quality and were included in the meta-analysis. Those with less than 12 points were excluded. Besides, if there was overlap between authors or centers, only the higher-quality or more recent literature was selected.

2.3. Data Extraction and Quality Assessment. Two researchers evaluated all the titles and abstracts. Then they assessed the selected full-text articles for eligibility. This work was then reevaluated and confirmed by a senior researcher. The measured outcomes of all eligible publications can be divided into two categories: (1) short-term outcomes (operation time, estimated blood loss, transfusion, length of hospital stay, morbidity, and mortality); (2) oncological outcomes (tumor size, margin distance, $\mathrm{R} 0$ resection, recurrence, and survival). The postoperative morbidity was cataloged according to the Clavien-Dindo classification. Minor complication refers to Grade I and Grade II complications, and major complication includes Grade III to V complications.

2.4. Subgroup Analysis. Because the different levels of hepatectomy can lead to different outcomes, and major hepatectomy is a technically dependent and time-consuming procedure, subgroup analyses were carried out according to surgical extensions. Included studies were assigned to 3 subgroups: minor hepatectomy, mixed hepatectomy, and major hepatectomy.

2.5. Statistical Analysis. The risk ratio (RR) was utilized to analyze the dichotomous variables, and the weighted mean difference (WMD) was utilized to assess the continuous variables. If the study provided medians and ranges instead of means and standard deviations (SDs), we estimated the means and SDs as described by Hozo et al. [12]. Heterogeneity was evaluated by Cochran's $Q$ statistic and Higgins $I^{2}$ statistic [13]. If data was not significantly heterogeneous $(P>0.05$ or $\left.I^{2}<50 \%\right)$, the pooled effects were calculated using a fixed model. Otherwise, the pooled effects were calculated using a random model. The hazard ratios (HRs) of a 5-year overall survival rate (OS) and a 5-year disease-free survival rate (DFS) were used with a generic inverse variance metaanalysis. The log HR and its SE were estimated using the method introduced by Tierney et al. [14]. According to the overall morbidity, potential publication bias was determined by carrying out an informal visual inspection of funnel plots. A two-tailed value of $P<0.05$ was considered significant. All statistical tests were performed with Review Manager version 5.1 (The Cochrane Collaboration, Oxford, England).

\section{Results}

3.1. Search Results and Baseline Characteristics. The last search was performed on September 20, 2017. A total of 968 potential published articles were initially identified from the search. Of these, 63 articles were selected based on their titles and abstracts, and a full examination of the texts was performed. Further 37 papers were excluded, after being read thoroughly, due to (1) including non-HCC cases $(n=4)$, (2) focusing on recurrent HCC $(n=3)$, (3) robot-assisted hepatectomy $(n=1)$, (4) overlap patient cohorts $(n=2)$, or (5) nonmatched comparative studies $(n=27)$. Then 26 studies were selected for quality assessment, and 6 studies were excluded by a modified MINORS score < 12 [15-20]. Finally, 20 studies were selected for final meta-analysis [2140]. A flow chart of the search strategies, which contains reasons for excluding studies, is elucidated in Figure 1. The details of the selection process, which included the references of excluded studies and the MINORS assessments of lowquality studies, could be found in Supplementary Materials (available here).

3.2. Study Characteristics. A total of 830 patients were included in the analysis with 388 undergoing $\mathrm{LH}$ (46.8\%) and 442 undergoing $\mathrm{OH}$ (53.2\%). The characteristics of these included studies are summarized in Table 1. Studies were well matched in terms of age, gender, ASA classification, body mass index (BMI), tumor size, and surgical extension. Eight studies reported only minor hepatectomy, and three studies focused on major hepatectomy, whereas the remaining nine studies included both minor and major hepatectomy. The majority of studies graded morbidity according to the Clavien-Dindo classification, with the study by Lee et al. being the only exception [24]. The assessments of the NRCTs are illustrated in Table 2. Each trial received more than 12 points (the maximum possible score is 16) and was considered to be of the highest quality (see Supplementary Materials). 
TABLE 1: Basic information of the included literature.

\begin{tabular}{|c|c|c|c|c|c|c|c|c|c|c|c|}
\hline \multirow{2}{*}{ Author } & \multirow{2}{*}{ Region } & \multirow{2}{*}{ Year } & \multirow{2}{*}{ Study period } & \multicolumn{2}{|c|}{ Sample size } & \multirow{2}{*}{$\begin{array}{l}\text { Matching } \\
\text { method }\end{array}$} & \multicolumn{2}{|c|}{ Cirrhosis (\%) } & \multirow{2}{*}{$\begin{array}{l}\text { Surgical } \\
\text { extension }\end{array}$} & \multirow{2}{*}{ Conversion (\%) } & \multirow{2}{*}{$\begin{array}{c}\text { Clavien- } \\
\text { Dindo }\end{array}$} \\
\hline & & & & LH & $\mathrm{OH}$ & & LH & $\mathrm{OH}$ & & & \\
\hline Belli et al. & Italy & 2007 & $2000-2004$ & 23 & 23 & $\mathrm{CCM}$ & 100 & 100 & Minor & 4.3 & Yes \\
\hline Tranchart et al. & France & 2010 & 1999-2008 & 42 & 42 & CCM & 73.8 & 81 & Mixed & 4.8 & Yes \\
\hline Kim et al. & Korea & 2011 & 2005-2009 & 26 & 29 & $\mathrm{CCM}$ & 92.3 & 86.2 & Mixed & $\mathrm{E}$ & Yes \\
\hline Truant et al. & France & 2011 & 2002-2009 & 36 & 53 & CCM & 100 & 100 & Minor & 19.4 & Yes \\
\hline Lee et al. & Hong Kong & 2011 & 2004-2010 & 33 & 50 & $\mathrm{CCM}$ & 84.8 & 64 & Minor & 18.2 & NA \\
\hline Ahn et al. & Korea & 2014 & 2005-2013 & 51 & 51 & PSM & 68.6 & 66.8 & Mixed & 9.8 & Yes \\
\hline Kim et al. & Korea & 2014 & $2000-2012$ & 29 & 29 & PSM & 62.1 & 65.5 & Minor & $\mathrm{E}$ & Yes \\
\hline Memeo et al. & France & 2014 & 1990-2009 & 45 & 45 & CCM & 100 & 100 & Minor & NA & Yes \\
\hline Lau et al. & USA & 2015 & 2008-2014 & 26 & 26 & $\mathrm{CCM}$ & 80.8 & 73.1 & Mixed & 35 & Yes \\
\hline Lee et al. & Canada & 2015 & 2006-2013 & 43 & 86 & $\mathrm{CCM}$ & NA & NA & Mixed & 14 & Yes \\
\hline Luo et al. & China & 2015 & 2008-2015 & 53 & 53 & $\mathrm{CCM}$ & 100 & 100 & Minor & $\mathrm{E}$ & Yes \\
\hline Takahara et al. & Japan & 2015 & 2000-2010 & 387 & 387 & PSM & 61.7 & 59.6 & Mixed & 6.5 & Yes \\
\hline Han et al. & Korea & 2015 & 2004-2013 & 88 & 88 & PSM & 62.5 & 59.1 & Mixed & 9.1 & Yes \\
\hline Yoon et al. & Korea & 2015 & 2007-2011 & 58 & 174 & PSM & NA & NA & Mixed & 0 & Yes \\
\hline Cheung et al. & Hong Kong & 2016 & 2002-2015 & 110 & 330 & PSM & 100 & 100 & Mixed & 5.5 & Yes \\
\hline Sposito et al. & Italy & 2016 & $2006-2013$ & 43 & 43 & PSM & 100 & 100 & Minor & 4.7 & Yes \\
\hline Jiang et al. & China & 2016 & 2008-2013 & 59 & 59 & PSM & 100 & 100 & Minor & 5.1 & Yes \\
\hline Komatsu et al. & France & 2016 & 2000-2014 & 38 & 38 & $\mathrm{CCM}$ & NA & NA & Major & 31.6 & Yes \\
\hline Yoon et al. & Korea & 2017 & 2008-2015 & 33 & 33 & PSM & 100 & 100 & Major & NA & Yes \\
\hline $\mathrm{Xu}$ et al. & China & 2017 & 2015-2017 & 32 & 32 & PSM & 100 & 100 & Major & NA & Yes \\
\hline
\end{tabular}

CCM: case by case matching; PSM: propensity score matching; E: conversion cases were excluded from the studies; NA: not available.

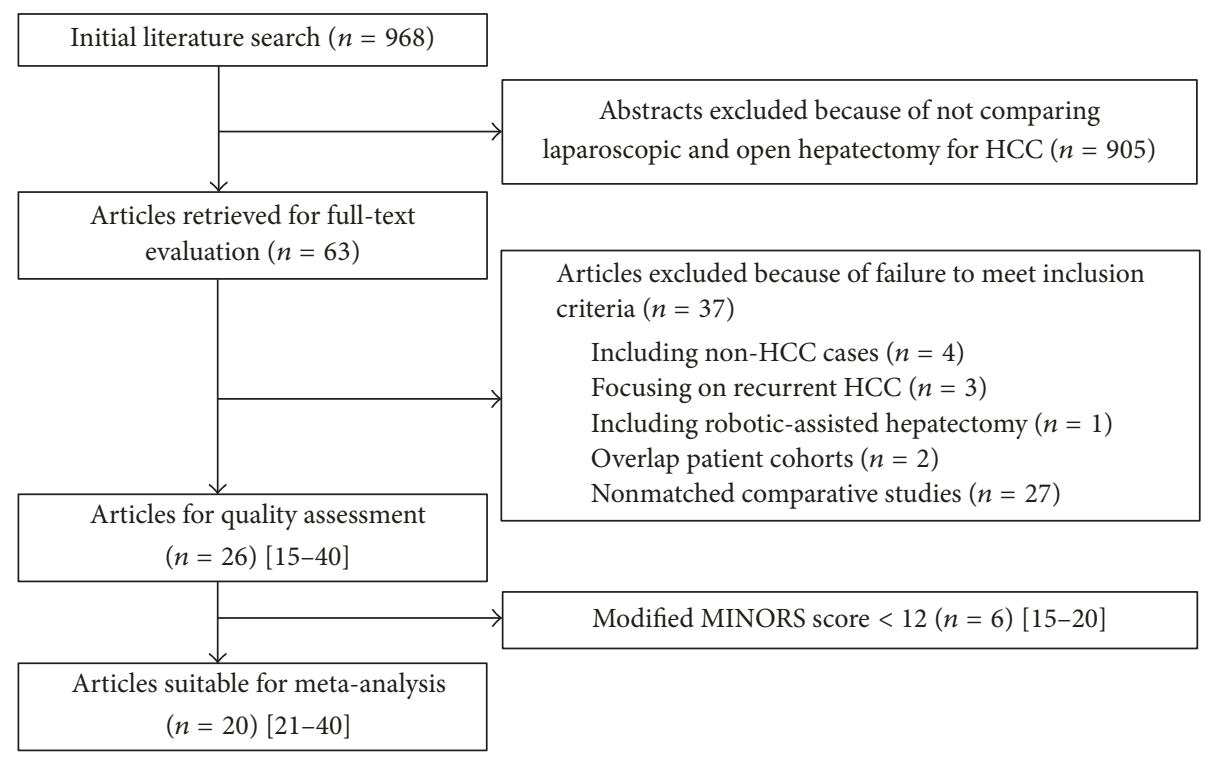

FIGURE 1: Flow chart of literature search strategies.

\subsection{Meta-Analysis of Short-Term Outcomes}

3.3.1. Operation Time. Operative time was reported in all studies [21-40]. Statistically significant between-study heterogeneity was identified in all subgroups $\left(P<0.01, I^{2}\right.$ $=87.2 \%)$. There was no significant difference between the groups' operation times (Table 3 ). However, in the subgroup of major hepatectomy, the overall effect size of the mean operation time was significantly longer in $\mathrm{LH}$ than that in $\mathrm{OH}$ (WMD $=77.93 \mathrm{~min}, 95 \% \mathrm{CI}: 40.45 \sim 115.41, P<0.01$ ).

3.3.2. Intraoperative Blood Loss. Blood loss was available from 17 studies [21, 22, 24-34, 36, 37, 39, 40]. Statistically significant between-study heterogeneity was identified in all subgroups $\left(P<0.01, I^{2}=88.1 \%\right)$. The pooled results showed that $\mathrm{LH}$ was associated with less blood loss than $\mathrm{OH}$ (Table 3). 
TABLE 2: Modified MINORS score of all eligible nonrandomized comparative studies.

\begin{tabular}{|c|c|c|c|c|c|c|c|c|c|}
\hline Author & (1) & (2) & (3) & (4) & (5) & (6) & (7) & (8) & Score \\
\hline Belli et al. & 2 & 2 & 1 & 2 & 2 & 1 & 2 & 1 & 13 \\
\hline Tranchart et al. & 2 & 2 & 1 & 1 & 2 & 1 & 2 & 2 & 13 \\
\hline Kim et al. & 2 & 1 & 1 & 2 & 2 & 2 & 1 & 1 & 12 \\
\hline Truant et al. & 2 & 2 & 1 & 1 & 2 & 2 & 1 & 1 & 12 \\
\hline Lee et al. & 2 & 2 & 1 & 1 & 2 & 2 & 1 & 1 & 12 \\
\hline Ahn et al. & 2 & 2 & 1 & 1 & 2 & 2 & 2 & 2 & 14 \\
\hline Kim et al. & 2 & 1 & 1 & 2 & 2 & 2 & 1 & 1 & 12 \\
\hline Memeo et al. & 2 & 1 & 1 & 1 & 2 & 1 & 2 & 2 & 12 \\
\hline Lau et al. & 2 & 1 & 2 & 1 & 2 & 2 & 2 & 1 & 13 \\
\hline Lee et al. & 2 & 1 & 1 & 1 & 2 & 2 & 1 & 2 & 12 \\
\hline Luo et al. & 2 & 1 & 1 & 1 & 2 & 2 & 2 & 2 & 13 \\
\hline Takahara et al. & 2 & 1 & 1 & 1 & 2 & 2 & 2 & 2 & 13 \\
\hline Han et al. & 2 & 1 & 1 & 1 & 2 & 2 & 2 & 2 & 13 \\
\hline Yoon et al. & 2 & 1 & 1 & 1 & 2 & 2 & 1 & 2 & 12 \\
\hline Cheung et al. & 2 & 2 & 1 & 1 & 2 & 2 & 2 & 2 & 14 \\
\hline Sposito et al. & 2 & 2 & 1 & 1 & 2 & 2 & 2 & 2 & 14 \\
\hline Jiang et al. & 2 & 1 & 1 & 1 & 2 & 2 & 2 & 2 & 13 \\
\hline Komatsu et al. & 2 & 1 & 1 & 1 & 2 & 2 & 2 & 1 & 12 \\
\hline Yoon et al. & 2 & 1 & 2 & 2 & 2 & 2 & 2 & 1 & 14 \\
\hline Xu et al. & 2 & 2 & 2 & 1 & 2 & 2 & 2 & 1 & 14 \\
\hline
\end{tabular}

(1) Consecutive patients, (2) prospective data collection, (3) reported endpoints, (4) unbiased outcome evaluation, (5) appropriate controls, (6) contemporary groups, (7) groups equivalent, (8) sample size.

However, in the subgroup of major hepatectomy, there was no significant difference between groups (WMD $=3.75 \mathrm{ml}, 95 \%$ CI: $-60.16 \sim 67.65, P=0.88)$.

3.3.3. Blood Transfusion. Fourteen studies recorded perioperative blood transfusion [21-27, 29, 30, 33-35, 37, 39]. There was no evidence of heterogeneity between subgroups $(P=$ $\left.0.81, I^{2}=0 \%\right)$. Although none of the subgroups reached a significant difference, the overall pooled data indicated that transfusion rates were lower in $\mathrm{LH}(\mathrm{RR}=0.73,95 \% \mathrm{CI}$ : $0.55 \sim 0.96, P=0.03$ ) (Table 3).

3.3.4. Duration of Hospital Stay. The length of hospital stays was pooled for all studies [21-40]. Although statistically significant between-study heterogeneity was identified in each subgroup, there was no evidence of heterogeneity between subgroups $\left(P=0.99, I^{2}=0 \%\right)$. Hospital stays in LH group were shorter than those in $\mathrm{OH}$ group (WMD $=-2.86 \mathrm{~d}, 95 \%$ CI: $-3.63 \sim-2.08, P<0.01$ ) (Table 3).

3.3.5. Morbidity. All studies reported their overall complication rates [21-40]. Because there was no statistical evidence of heterogeneity, the effect sizes of all subgroups were synthesized to generate the overall effect size $\left(P=0.91, I^{2}=\right.$ $0 \%$ ) (Table 3) (Figure 2). The postoperative morbidity rates were $14.0 \%(176 / 1255)$ in $\mathrm{LH}$ and $24.2 \%$ (404/1671) in $\mathrm{OH}$. In addition the pooled data showed that LH significantly reduced postoperative complications $(\mathrm{RR}=0.55 ; 95 \% \mathrm{CI}$, $0.47 \sim 0.65 ; P<0.01$ ) (Table 3). Moreover, each subgroup also revealed reduced overall morbidity in the LH group (Table 3).
Eighteen studies recorded severe complications [21, 22, 25-40]. Similar to overall morbidity, the results showed that patients in the LH group suffered less severe complications (Table 3). We identified specified complications of ascites, liver failure, and the respiratory system. The results implied that postoperative ascites in patients, regardless of whether they underwent minor or major hepatectomy, was less in $\mathrm{LH}$ than in $\mathrm{OH}(\mathrm{RR}=0.42 ; 95 \% \mathrm{CI}, 0.31 \sim 0.59 ; P<0.01)$ (Figure 3(a)). Studies that recorded postoperative liver failure reported a lower incidence of liver failure in $\mathrm{LH}$ than in $\mathrm{OH}$ with one exception by $\mathrm{Xu}$ et al. [39]. The overall pooled data revealed that patients in the LH group were less likely to suffer liver failure than those in the $\mathrm{OH}$ group $(\mathrm{RR}=$ 0.41; 95\% CI, 0.27 0.64; $P<0.01$ ) (Figure 3(b)). LH was also associated with a significant reduction in respiratory complications regardless of different surgical extension (RR $=0.43,95 \%$ CI: $0.28 \sim 0.64, P<0.01)$ (Figure 3(c)).

3.3.6. Mortality. Nine studies recorded cases of postoperative death $[21,22,25,28-30,33,35,39]$. There was no evidence of heterogeneity between subgroups $\left(P=0.97, I^{2}=0 \%\right)$. These studies showed very low incidences of mortality. However, the overall pooled data indicated a more reduced postoperative mortality in $\mathrm{LH}$ than that in $\mathrm{OH}(\mathrm{RR}=0.43$; 95\% CI, 0.18 1.00; $P=0.05$ ) (Table 3).

\subsection{Meta-Analysis of Oncological Outcomes}

3.4.1. Tumor Size. Only one study did not report tumor size [30]. There was trifling heterogeneity between subgroups, mainly due to the major hepatectomy subgroup $\left(P=0.35, I^{2}\right.$ 
TABLE 3: Overall outcomes of the meta-analysis.

\begin{tabular}{|c|c|c|c|c|c|c|c|c|}
\hline \multirow{2}{*}{ Outcomes } & \multirow{2}{*}{$\begin{array}{l}\text { Studies } \\
\text { No. }\end{array}$} & \multicolumn{2}{|c|}{ Sample size } & \multirow{2}{*}{$\begin{array}{l}\text { Heterogeneity } \\
\qquad\left(P, I^{2}\right)\end{array}$} & \multirow{2}{*}{ Model } & \multirow{2}{*}{ Overall effect size } & \multirow{2}{*}{$\begin{array}{c}\text { 95\% CI of overall } \\
\text { effect }\end{array}$} & \multirow{2}{*}{$P$} \\
\hline & & $\mathrm{LH}$ & $\mathrm{OH}$ & & & & & \\
\hline Operation time (min) & 20 & 1255 & 1671 & $<0.01,87 \%$ & $\mathrm{R}$ & $\mathrm{WMD}=9.15$ & $-7.61 \sim 25.90$ & 0.28 \\
\hline Minor hepatectomy & 8 & 321 & 355 & $<0.01,78 \%$ & $\mathrm{R}$ & $\mathrm{WMD}=12.04$ & $-5.31 \sim 29.39$ & 0.17 \\
\hline Mixed hepatectomy & 9 & 831 & 1213 & $<0.01,83 \%$ & $\mathrm{R}$ & $\mathrm{WMD}=-14.28$ & $-40.76 \sim 12.21$ & 0.29 \\
\hline Major hepatectomy & 3 & 103 & 103 & $0.05,67 \%$ & $\mathrm{R}$ & $\mathrm{WMD}=77.93$ & $40.45 \sim 115.41$ & $<0.01$ \\
\hline Blood loss $(m L)$ & 17 & 1128 & 1425 & $<0.01,92 \%$ & $\mathrm{R}$ & $\mathrm{WMD}=-93.21$ & $-157.33 \sim-29.09$ & $<0.01$ \\
\hline Minor hepatectomy & 7 & 278 & 312 & $0.39,5 \%$ & $\mathrm{R}$ & $\mathrm{WMD}=-76.21$ & $-98.41 \sim-54.01$ & $<0.01$ \\
\hline Mixed hepatectomy & 7 & 747 & 1010 & $0.05,52 \%$ & $\mathrm{R}$ & $\mathrm{WMD}=-212.94$ & $-294.57 \sim-131.31$ & $<0.01$ \\
\hline Major hepatectomy & 3 & 103 & 103 & $0.88,0 \%$ & $\mathrm{R}$ & $\mathrm{WMD}=3.75$ & $-60.16 \sim 67.65$ & 0.88 \\
\hline Transfusion & 14 & 979 & 1352 & $0.90,0 \%$ & $\mathrm{~F}$ & $\mathrm{RR}=0.73$ & $0.55 \sim 0.96$ & 0.03 \\
\hline Minor hepatectomy & 4 & 121 & 155 & $0.49,0 \%$ & $\mathrm{~F}$ & $\mathrm{RR}=0.53$ & $0.19 \sim 1.45$ & 0.22 \\
\hline Mixed hepatectomy & 8 & 788 & 1127 & $0.86,0 \%$ & $\mathrm{~F}$ & $\mathrm{RR}=0.75$ & $0.55 \sim 1.01$ & 0.06 \\
\hline Major hepatectomy & 2 & 70 & 70 & $0.28,15 \%$ & $\mathrm{~F}$ & $\mathrm{RR}=0.75$ & $0.17 \sim 3.25$ & 0.70 \\
\hline Hospital stay (days) & 20 & 1255 & 1671 & $<0.01,80 \%$ & $\mathrm{R}$ & $\mathrm{WMD}=-2.86$ & $-3.63 \sim-2.08$ & $<0.01$ \\
\hline Minor hepatectomy & 8 & 321 & 355 & $<0.01,76 \%$ & $\mathrm{R}$ & $\mathrm{WMD}=-2.93$ & $-4.23 \sim-1.63$ & $<0.01$ \\
\hline Mixed hepatectomy & 9 & 831 & 1213 & $0.01,58 \%$ & $\mathrm{R}$ & $\mathrm{WMD}=-2.85$ & $-3.95 \sim-1.76$ & $<0.01$ \\
\hline Major hepatectomy & 3 & 103 & 103 & $0.15,47 \%$ & $\mathrm{R}$ & $\mathrm{WMD}=-2.76$ & $-4.60 \sim-0.92$ & $<0.01$ \\
\hline Morbidity & 20 & 1255 & 1671 & $0.28,14 \%$ & $\mathrm{~F}$ & $\mathrm{RR}=0.55$ & $0.47 \sim 0.65$ & $<0.01$ \\
\hline Minor hepatectomy & 8 & 321 & 355 & $0.29,17 \%$ & $\mathrm{~F}$ & $\mathrm{RR}=0.53$ & $0.41 \sim 0.69$ & $<0.01$ \\
\hline Mixed hepatectomy & 9 & 831 & 1213 & $0.26,21 \%$ & $\mathrm{~F}$ & $\mathrm{RR}=0.57$ & $0.46 \sim 0.72$ & $<0.01$ \\
\hline Major hepatectomy & 3 & 103 & 103 & $0.21,37 \%$ & $\mathrm{~F}$ & $\mathrm{RR}=0.55$ & $0.36 \sim 0.83$ & $<0.01$ \\
\hline Severe complications & 18 & 1196 & 1476 & $0.88,0 \%$ & $\mathrm{~F}$ & $\mathrm{RR}=0.51$ & $0.39 \sim 0.68$ & $<0.01$ \\
\hline Minor hepatectomy & 7 & 288 & 305 & $0.96,0 \%$ & $\mathrm{~F}$ & $\mathrm{RR}=0.48$ & $0.24 \sim 0.96$ & 0.04 \\
\hline Mixed hepatectomy & 8 & 805 & 1068 & $0.44,0 \%$ & $\mathrm{~F}$ & $\mathrm{RR}=0.54$ & $0.38 \sim 0.76$ & $<0.01$ \\
\hline Major hepatectomy & 3 & 103 & 103 & $0.35,6 \%$ & $\mathrm{~F}$ & $\mathrm{RR}=0.42$ & $0.18 \sim 1.00$ & 0.05 \\
\hline Mortality & 9 & 789 & 1026 & $0.88,0$ & $\mathrm{~F}$ & $\mathrm{RR}=0.43$ & $0.18 \sim 1.00$ & 0.05 \\
\hline Minor hepatectomy & 3 & 104 & 121 & $0.34,7$ & $\mathrm{~F}$ & $\mathrm{RR}=0.39$ & $0.09 \sim 1.68$ & 0.21 \\
\hline Mixed hepatectomy & 5 & 653 & 873 & $0.84,0$ & $\mathrm{~F}$ & $\mathrm{RR}=0.46$ & $0.15 \sim 1.43$ & 0.18 \\
\hline Major hepatectomy & 1 & 32 & 32 & Not applicable & $\mathrm{F}$ & $\mathrm{RR}=0.33$ & $0.01 \sim 7.89$ & 0.50 \\
\hline Tumor size $(\mathrm{cm})$ & 19 & 1229 & 1645 & $<0.01,57 \%$ & $\mathrm{R}$ & $\mathrm{WMD}=-0.19$ & $-0.41 \sim 0.03$ & 0.09 \\
\hline Minor hepatectomy & 8 & 321 & 355 & $0.76,0 \%$ & $\mathrm{R}$ & $\mathrm{WMD}=-0.07$ & $-0.26 \sim 0.12$ & 0.48 \\
\hline Mixed hepatectomy & 8 & 805 & 1187 & $0.50,0 \%$ & $\mathrm{R}$ & $\mathrm{WMD}=-0.09$ & $-0.25 \sim 0.07$ & 0.28 \\
\hline Major hepatectomy & 3 & 103 & 103 & $<0.01,92 \%$ & $\mathrm{R}$ & $\mathrm{WMD}=-1.77$ & $-4.06 \sim 0.53$ & 0.13 \\
\hline Margin distance $(\mathrm{cm})$ & 11 & 501 & 694 & $0.14,47 \%$ & $\mathrm{R}$ & $\mathrm{WMD}=2.61$ & $1.06 \sim 4.17$ & $<0.01$ \\
\hline Minor hepatectomy & 5 & 186 & 220 & $0.06,56 \%$ & $\mathrm{R}$ & $\mathrm{WMD}=2.16$ & $0.15 \sim 4.17$ & 0.03 \\
\hline Mixed hepatectomy & 5 & 282 & 441 & $0.15,41 \%$ & $\mathrm{R}$ & $\mathrm{WMD}=3.20$ & $0.41 \sim 5.99$ & 0.02 \\
\hline Major hepatectomy & 1 & 33 & 33 & Not applicable & $\mathrm{R}$ & $\mathrm{WMD}=5.90$ & $-2.69 \sim 14.49$ & 0.18 \\
\hline R0 resection & 14 & 1010 & 1409 & $0.70,0 \%$ & $\mathrm{~F}$ & $\mathrm{RR}=1.01$ & $0.99 \sim 1.02$ & 0.37 \\
\hline Minor hepatectomy & 6 & 240 & 257 & $0.80,0 \%$ & $\mathrm{~F}$ & $\mathrm{RR}=0.98$ & $0.95 \sim 1.01$ & 0.23 \\
\hline Mixed hepatectomy & 7 & 738 & 1120 & $0.47,0 \%$ & $\mathrm{~F}$ & $\mathrm{RR}=1.01$ & $1.00 \sim 1.03$ & 0.13 \\
\hline Major hepatectomy & 1 & 32 & 32 & Not applicable & $\mathrm{F}$ & $\mathrm{RR}=1.03$ & $0.93 \sim 1.15$ & 0.56 \\
\hline
\end{tabular}

WMD: weighted mean difference; RR: risk ratio; F: fixed; R: random.

$=4.2 \%$ ) (Table 3 ). Meta-analysis showed that the tumor size of $\mathrm{OH}$ was longer than that of $\mathrm{LH}$ with a marginal difference (WMD $=-0.19 \mathrm{~cm} ; 95 \% \mathrm{CI}:-0.41 \sim-0.03, P=0.09$ ), which was mainly due to smaller tumors in $\mathrm{LH}$ than those in $\mathrm{OH}$ in the major hepatectomy subgroup (Table 3 ).
3.4.2. Margin Distance. Only 11 studies mentioned the distance of the tumor margin $[22,24-29,31,34,38,40]$. Although statistical significant between-study heterogeneity was identified in each subgroup, there was no evidence of heterogeneity between subgroups $\left(P=0.63, I^{2}=0 \%\right)$. On 


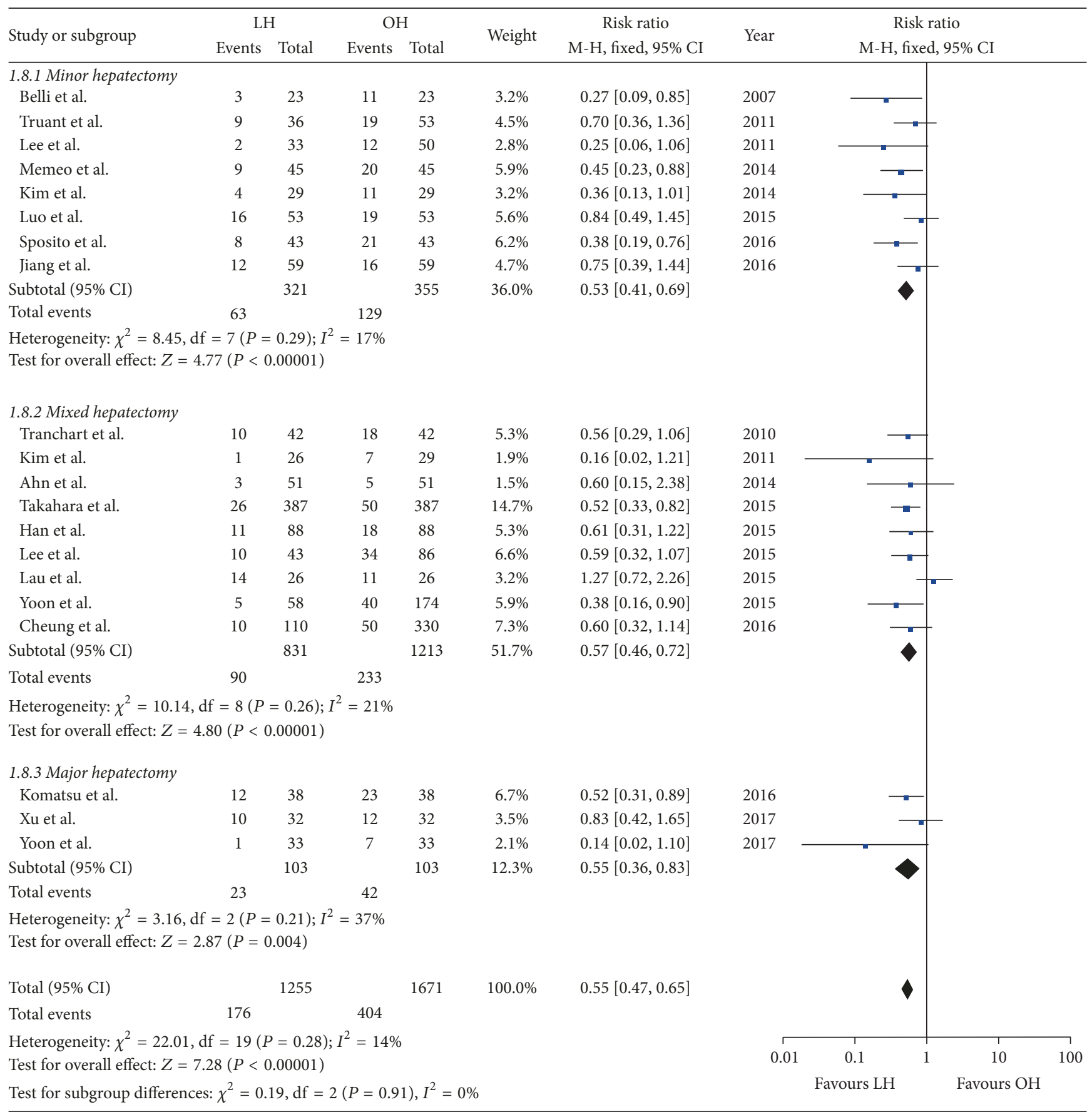

FIGURE 2: Forest plot of overall morbidity.

pooling the results, the margin distance was longer in the $\mathrm{LH}$ group than that in the $\mathrm{OH}$ group $(\mathrm{WMD}=2.61 \mathrm{~cm} ; 95 \% \mathrm{CI}$ : 1.06 4.17, $P<0.01$ ) (Table 3).

3.4.3. R0 Resection. The $\mathrm{R} 0$ resection was reported in 14 studies [21, 23, 24, 27, 29-36, 38, 39]. There was no obvious heterogeneity $\left(P=0.18, I^{2}=41.8 \%\right)$. The pooled estimate for margin distance indicated comparative outcomes between groups $(\mathrm{RR}=1.01,95 \% \mathrm{CI}: 0.99 \sim 1.02, P=0.37)$ (Table 3).
3.4.4. Overall Survival Rate and Disease-Free Survival Rate. Summary of follow-up time, recurrence, and long-term survival rates is listed in Table 4 . Nineteen studies reported the detailed long-term outcomes. Among them, the data for 5 -year OS rates can be extracted from nine studies and the data for 5-year DFS rates can be extracted from ten studies. The follow-up periods in six studies were less than five years. The survival data of three studies cannot be extracted due to a technical problem with figures. Unfortunately, none of the three major hepatectomy studies can be included in our survival analysis $[37,39,40]$. In all, the pooled 5-year OS rate 


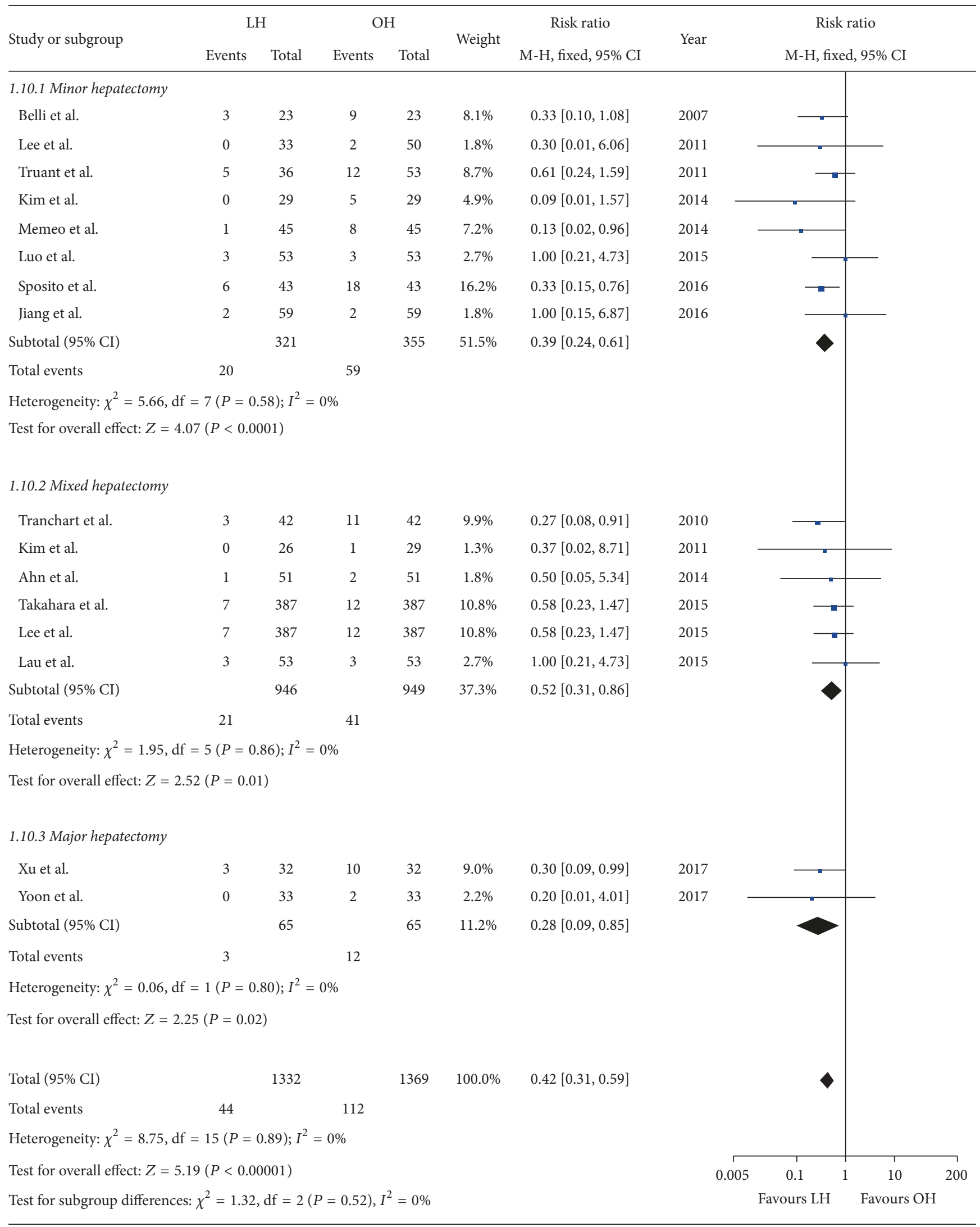

(a)

Figure 3: Continued. 


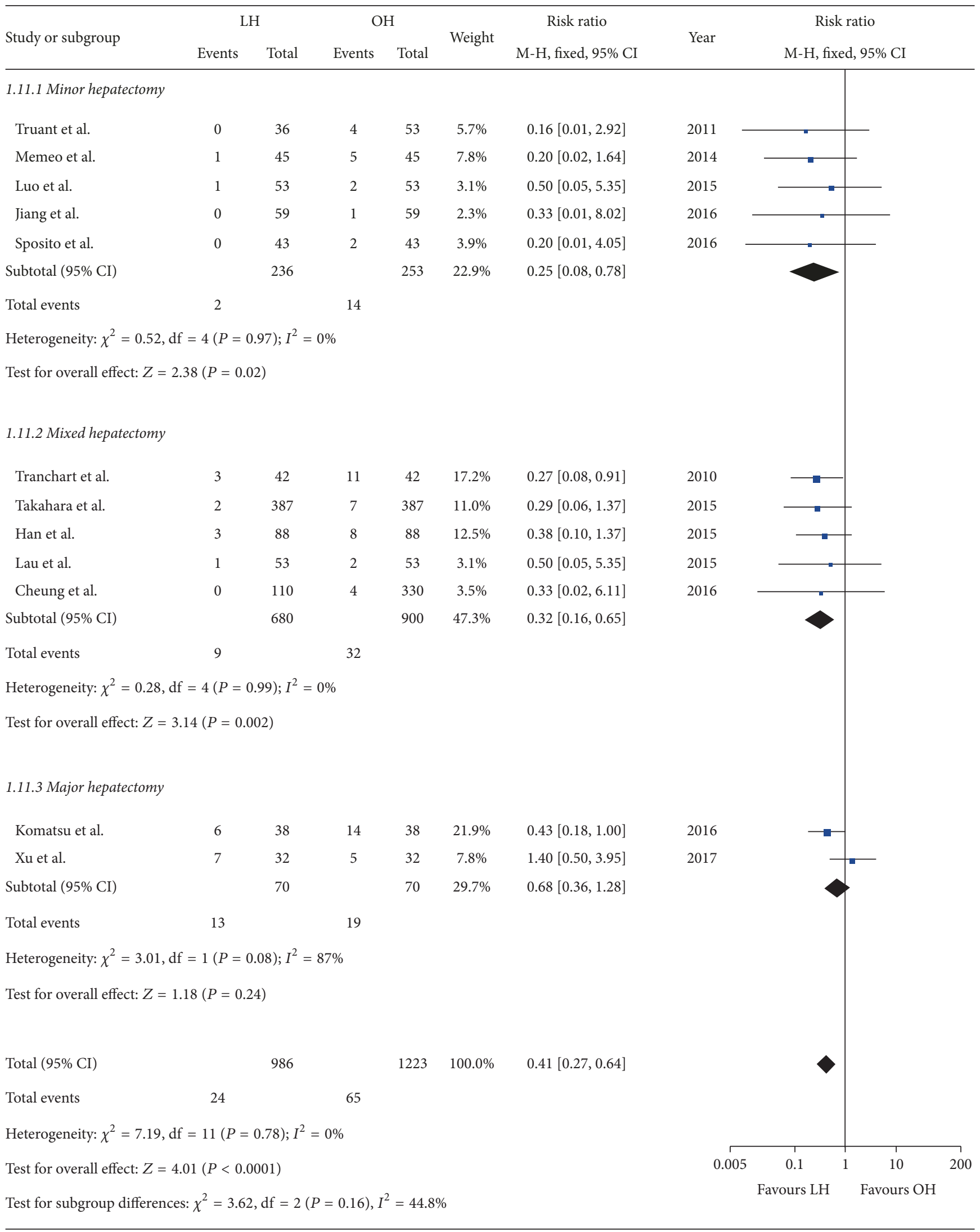

(b)

Figure 3: Continued. 


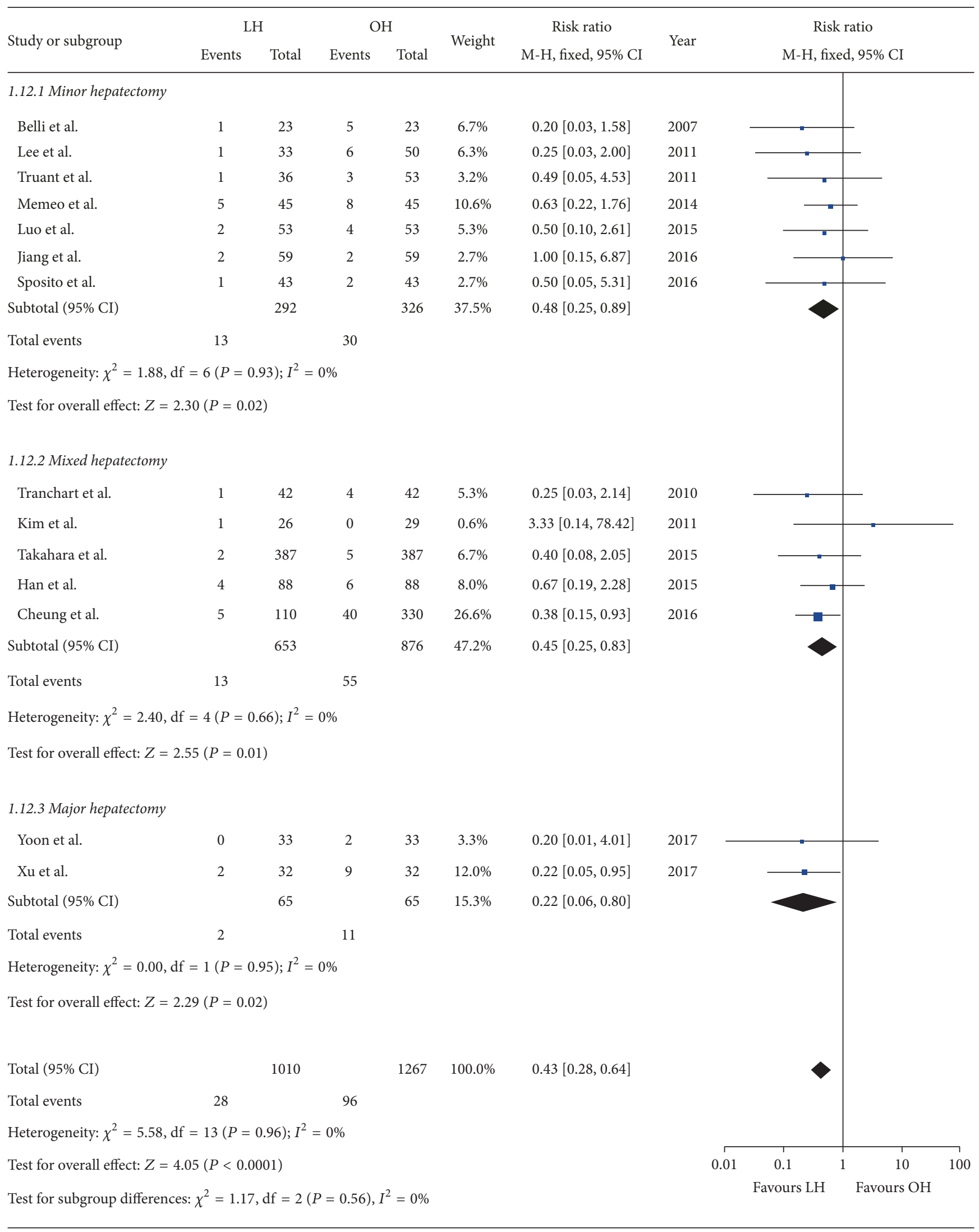

(c)

FIGURE 3: Forest plot of specific complications: (a) ascites, (b) liver failure, (c) respiratory complications. 
TABLE 4: Summary of recurrence and long-term survival.

\begin{tabular}{|c|c|c|c|c|}
\hline Author & Group & Follow-up & $\mathrm{R}$ & Survival (time: month; rate: \%) \\
\hline \multirow{2}{*}{ Tranchart et al. } & $\mathrm{LH}$ & 29.7 & 10 & 1, 3, 5 y-DFS: 81.6, 60.9, 45.6; 1, 3, 5 y-OS: 93.1, 74.4, 59.5. \\
\hline & $\mathrm{OH}$ & 24.6 & 12 & 1, 3, 5 y-DFS: 70.2, 54.3, 37.2; 1, 3, 5 y-OS: 81.8, 73, 47.4 \\
\hline \multirow{2}{*}{ Kim et al. } & $\mathrm{LH}$ & 21.8 & 7 & MDFS: 13.4; 1y-DFS: 84.6 \\
\hline & $\mathrm{OH}$ & 24.8 & 10 & MDFS: $14.6 ; 1$ y-DFS: 82.8. \\
\hline \multirow{2}{*}{ Truant et al. } & $\mathrm{LH}$ & \multirow{2}{*}{35.7} & 16 & 5 y-DFS: 35.5; 5 y-OS: 70 \\
\hline & $\mathrm{OH}$ & & 23 & 5 y-DFS: 33.6; 5 y-OS: 46 \\
\hline \multirow{2}{*}{ Lee et al. } & $\mathrm{LH}$ & 35.4 & 15 & 1, 3, 5 y-DFS: $78.8,51,45.3 ; 1,3,5 y$-OS: 86.9, 81.8, 76.0. \\
\hline & $\mathrm{OH}$ & 28.5 & 19 & 1, 3, 5 y-DFS: $69.2,55.9,55.9 ; 1,3,5$ y-OS: $98,80.6,76.1$ \\
\hline \multirow{2}{*}{ Ahn et al. } & $\mathrm{LH}$ & 38.6 & 12 & 5 y-DFS: $67.8 ; 5$ y-OS: 80.1 \\
\hline & $\mathrm{OH}$ & 52.3 & 21 & 5 y-DFS: $54.8 ; 5$ y-OS: 85.7 \\
\hline \multirow{2}{*}{ Kim et al. } & $\mathrm{LH}$ & 47.9 & 11 & MDFS: 15.4; MOS: 47.9; 1, 3, 5 y-DFS: 81.1, 61.7, 54.0; 1, 3, 5 y-OS: 100, 100, 92.2. \\
\hline & $\mathrm{OH}$ & 59.5 & 16 & MDFS: 32.6; MOS: 59.5; 1, 3, 5 y-DFS: 78.6, 60.9, 40.1; 1, 3, 5 y-OS: 96.5, 92.2, 87.7. \\
\hline \multirow{2}{*}{ Memeo et al. } & $\mathrm{LH}$ & NR & 25 & $1,5,10$ y-DFS: $80,19,0 ; 1,5,10$ y-OS: $88,59,12$ \\
\hline & $\mathrm{OH}$ & NR & 28 & 1, 5, 10 y-DFS: 60, 23, 9; 1, 5, 10 y-OS: 63, 44, 22. \\
\hline \multirow{2}{*}{ Lee et al. } & $\mathrm{LH}$ & 22.7 & NR & 1, 3, 5 y-DFS: $60.5,53.5,53.5 ; 1,3,5 y$-OS: 95.3, 89.7, 89.7. \\
\hline & $\mathrm{OH}$ & 44.4 & NR & 1, 3, 5 y-DFS: 81.5, 66.7, 58.6; 1, 3, 5y-OS: 93.9, 89.5, 87.3. \\
\hline \multirow{2}{*}{ Luo et al. } & $\mathrm{LH}$ & 35 & 20 & MDFS: 21 \\
\hline & $\mathrm{OH}$ & 37 & 24 & MDFS: 18. \\
\hline \multirow{2}{*}{ Takahara et al. } & $\mathrm{LH}$ & 46.7 & NR & 1, 3, 5 y-DFS: 83.7, 58.3, 40.7; 1, 3, 5 y-OS: 95.8, 86.2, 76.8. \\
\hline & $\mathrm{OH}$ & 51.7 & NR & 1, 3, 5 y-DFS: 79.6, 50.4, 39.3; 1, 3, 5 y-OS: 95.8, 84.0, 70.9 \\
\hline \multirow{2}{*}{ Han et al. } & $\mathrm{LH}$ & 44.0 & 43 & 1, 3, 5 y-DFS: 69.7, 52.0, 44.2; 1, 3, 5 y-OS: 91.6, 87.5, 76.4. \\
\hline & $\mathrm{OH}$ & 48.7 & 46 & 1, 3, 5 y-DFS: 74.7, 49.5, 41.2; 1, 3, 5 y-OS: 93.1, 87.8, 73.2. \\
\hline \multirow{2}{*}{ Yoon et al. } & $\mathrm{LH}$ & NR & 16 & 1, 2, 3, 4 y-DFS: 82.0, 63.0, 56.0, 56.0; 1, 2, 3, 4 y-OS: 95.0, 92.0, 86.0, 86.0. \\
\hline & $\mathrm{OH}$ & NR & 31 & 1, 2, 3, 4 y-DFS: 88.0, 79.0, 62.0, 62.0; 1, 2, 3, 4 y-OS: 98.0, 93.0, 84.0, 68.0. \\
\hline \multirow{2}{*}{ Cheung et al. } & $\mathrm{LH}$ & 34.6 & 36 & MDFS: 66.4; MOS: 136; 1, 3, 5 y-DFS: 87.7, 65.8, 52.2; 1, 3, 5 y-OS: 98.9, 89.8, 83.7. \\
\hline & $\mathrm{OH}$ & 46.6 & 160 & MDFS: 52.4; MOS: $120 ; 1,3,5 \mathrm{y}$-DFS: $75.2,56.3,47.9 ; 1,3,5 \mathrm{y}$-OS: $94,79.3$, and 67.4. \\
\hline \multirow{2}{*}{ Sposito et al. } & $\mathrm{LH}$ & 39.3 & NR & MDFS: 25.5; MOS: 48.8; 3, 5 y-DFS: 41, 25; 3, 5 y-OS: 75, 38 \\
\hline & $\mathrm{OH}$ & 44.5 & NR & MDFS: 31.7; MOS: 57.8; 3, 5 y-DFS: 44, 11; 3, 5 y-OS: 79, 46. \\
\hline \multirow{2}{*}{ Jiang et al. } & $\mathrm{LH}$ & NR & 26 & MDFS: 17; 5 y-DFS: 44 \\
\hline & $\mathrm{OH}$ & NR & 30 & MDFS: 15; 5 y-DFS: 40. \\
\hline \multirow{2}{*}{ Komatsu et al. } & $\mathrm{LH}$ & 247 & NR & 3 y-DFS: $50.3 ; 3$ y-OS: 73.4 \\
\hline & $\mathrm{OH}$ & & NR & 3 y-DFS: $29.7 ; 3$ y-OS: 69.2 \\
\hline \multirow{2}{*}{ Yoon et al. } & $\mathrm{LH}$ & NR & NR & 2 y-DFS: 85.1; 2 y-OS: 100 \\
\hline & $\mathrm{OH}$ & NR & NR & 2 y-DFS: $83.9 ; 2$ y-OS: 88.8 \\
\hline \multirow{2}{*}{ Xu et al. } & $\mathrm{LH}$ & \multirow{2}{*}{13.8} & NR & 1, 2 y-DFS: 95.5, 72.9; 1, 2 y-OS: 100, 85.7. \\
\hline & $\mathrm{OH}$ & & NR & 1, 2 y-DFS: $93.5,81.5 ; 1,2$ y-OS: 96.3, 86.7. \\
\hline
\end{tabular}

Follow-up was shown as median month; R: recurrence; DFS: disease-free survival rate; OS: overall survival rate; MDFS: median disease-free survival time; MOS: median overall survival time; y: year; NR: not reported.

was slightly better in $\mathrm{LH}$ than in $\mathrm{OH}(\mathrm{HR}=0.66,95 \% \mathrm{CI}$ : $0.52 \sim 0.84, P<0.01$ ) (Figure 4(a)). The 5-year DFS rate was comparable between groups $(\mathrm{HR}=0.88,95 \% \mathrm{CI}: 0.74 \sim 1.06$, $P=0.18$ ) (Figure 4(b)).

3.4.5. Publication Bias. The study by Lau et al. was outside the funnel [30], and the remaining representative plots were distributed symmetrically. We believed such publication bias was acceptable in the studies (Figure 5).

\section{Discussion}

This meta-analysis selected and summarized high-quality literature that compared the short- and long-term outcomes of $\mathrm{LH}$ and $\mathrm{OH}$ for the treatment of HCC. All of the studies had case-matched design and were of high quality according to the modified MINORS scale. For short-term surgical outcomes, LH exhibited advantages in terms of blood loss, hospital stay, overall postoperative morbidity, and mortality, whereas no statistically significant differences were identified regarding operation time. As for oncological outcomes, R0 and survival rates of $\mathrm{LH}$ were also not inferior to $\mathrm{OH}$.

To date, there have been several meta-analyses comparing $\mathrm{LH}$ to $\mathrm{OH}$ for HCC [5-8]. The results have demonstrated that $\mathrm{LH}$ is comparable to $\mathrm{OH}$ regarding the operation time and postoperative mortality and is associated with less blood loss, as well as a shorter hospital stay (Table 5). Previous 


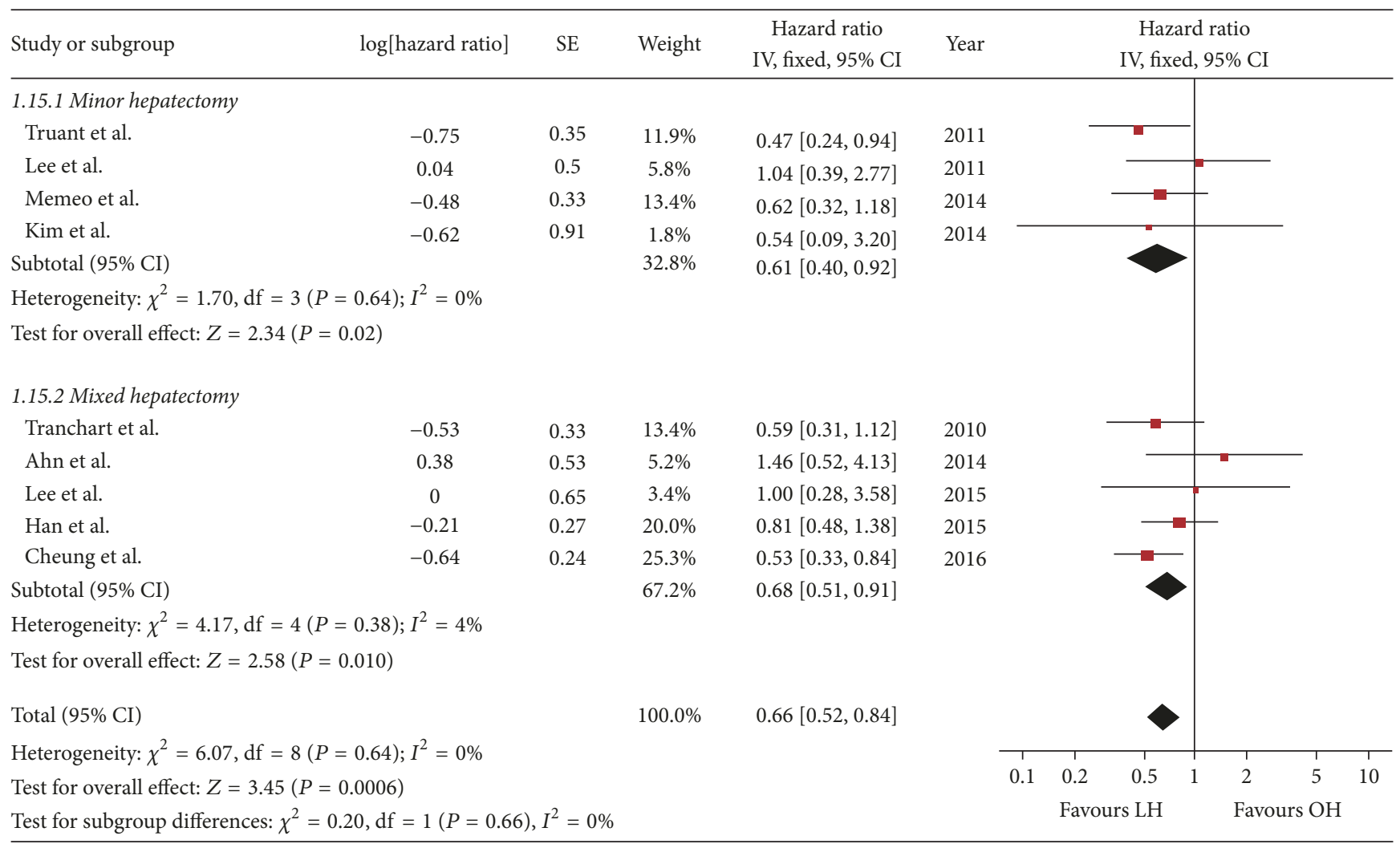

(a)

\begin{tabular}{|c|c|c|c|c|c|c|c|}
\hline Study or subgroup & $\log [$ hazard ratio $]$ & SE & Weight & $\begin{array}{c}\text { Hazard ratio } \\
\text { IV, fixed, 95\% CI }\end{array}$ & Year & \multicolumn{2}{|c|}{$\begin{array}{l}\text { Hazard ratio } \\
\text { IV, fixed, } 95 \% \text { CI }\end{array}$} \\
\hline \multicolumn{8}{|c|}{ 1.16.1 Minor hepatectomy } \\
\hline Lee et al. & 0.08 & 0.34 & $7.3 \%$ & $1.08[0.56,2.11]$ & 2011 & & \\
\hline Truant et al. & 0.15 & 0.28 & $10.7 \%$ & $1.16[0.67,2.01]$ & 2011 & & - \\
\hline Memeo et al. & -0.17 & 0.27 & $11.5 \%$ & $0.84[0.50,1.43]$ & 2014 & & \\
\hline Kim et al. & -0.28 & 0.36 & $6.5 \%$ & $0.76[0.37,1.53]$ & 2014 & & \\
\hline Subtotal $(95 \% \mathrm{CI})$ & & & $36.0 \%$ & $0.96[0.71,1.29]$ & & & \\
\hline \multicolumn{8}{|c|}{ Heterogeneity: $\chi^{2}=1.26, \mathrm{df}=3(P=0.74) ; I^{2}=0 \%$} \\
\hline \multicolumn{8}{|c|}{ Test for overall effect: $Z=0.29(P=0.77)$} \\
\hline \multicolumn{8}{|c|}{ 1.16.2 Mixed hepatectomy } \\
\hline Tranchart et al. & -0.33 & 0.29 & $10.0 \%$ & $0.72[0.41,1.27]$ & 2010 & & - \\
\hline Kim et al. & -0.15 & 0.43 & $4.5 \%$ & $0.86[0.37,2.00]$ & 2011 & & \\
\hline Ahn et al. & -0.38 & 0.32 & $8.2 \%$ & $0.68[0.37,1.28]$ & 2014 & & - \\
\hline Han et al. & 0 & 0.4 & $5.3 \%$ & $1.00[0.46,2.19]$ & 2015 & & \\
\hline Lee et al. & 0.63 & 0.35 & $6.9 \%$ & $1.88[0.95,3.73]$ & 2015 & & 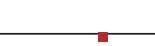 \\
\hline Cheung et al. & -0.27 & 0.17 & $29.1 \%$ & $0.76[0.55,1.07]$ & 2016 & & \\
\hline Subtotal $(95 \%$ CI $)$ & & & $64.0 \%$ & $0.85[0.68,1.06]$ & & & \\
\hline \multicolumn{8}{|c|}{ Heterogeneity: $\chi^{2}=6.49, \mathrm{df}=5(P=0.26) ; I^{2}=23 \%$} \\
\hline \multicolumn{8}{|c|}{ Test for overall effect: $Z=1.45(P=0.15)$} \\
\hline Total $(95 \%$ CI $)$ & & & $100.0 \%$ & $0.88[0.74,1.06]$ & & & \\
\hline \multicolumn{7}{|c|}{ Heterogeneity: $\chi^{2}=8.16, \mathrm{df}=9(P=0.52) ; I^{2}=0 \%$} & $\begin{array}{ll}1 & 1 \\
1.5 & 2\end{array}$ \\
\hline \multicolumn{6}{|c|}{$\begin{array}{l}\text { Test for overall effect: } Z=1.33(P=0.18) \\
\text { Test for subgroup differences: } \chi^{2}=0.41, \mathrm{df}=1(P=0.52), I^{2}=0 \%\end{array}$} & Favours LH & Favours $\mathrm{OH}$ \\
\hline
\end{tabular}

(b)

FIGURE 4: Forest plot of survival rate: (a) 5-year OS, (b) 5-year DFS. 
TABle 5: Previous meta-analyses comparing LH to OH for HCC.

\begin{tabular}{|c|c|c|c|c|}
\hline Variables & Zhou & $\mathrm{Li}$ & Xiong & Yin \\
\hline Year & 2011 & 2012 & 2012 & 2013 \\
\hline Included studies & 10 & 10 & 9 & 15 \\
\hline Total LH numbers & 213 & 244 & 234 & 485 \\
\hline Surgical extension & Minor resection & Minor resection & Minor resection & Minor resection \\
\hline Operation time & NS & NS & NS & NS \\
\hline Blood loss & Favor LH & Favor LH & Favor LH & Favor LH \\
\hline Overall morbidity & Favor LH & Favor LH & N/A & Favor LH \\
\hline Severe complications & N/A & N/A & N/A & N/A \\
\hline ascites & N/A & N/A & Favor LH & N/A \\
\hline Liver failure & NS & N/A & Favor LH & N/A \\
\hline Respiratory complications & NS & N/A & NS & N/A \\
\hline Mortality & NS & N/A & NS & $\mathrm{N} / \mathrm{A}$ \\
\hline Hospital stay & Favor LH & Favor LH & Favor LH & Favor LH \\
\hline Tumor size & N/A & N/A & N/A & N/A \\
\hline Margin distance & NS & NS & N/A & NS \\
\hline R0 resection & N/A & NS & NS & NS \\
\hline Survival & N/A & N/A & N/A & NS \\
\hline
\end{tabular}

NS: not significant, N/A: not available.

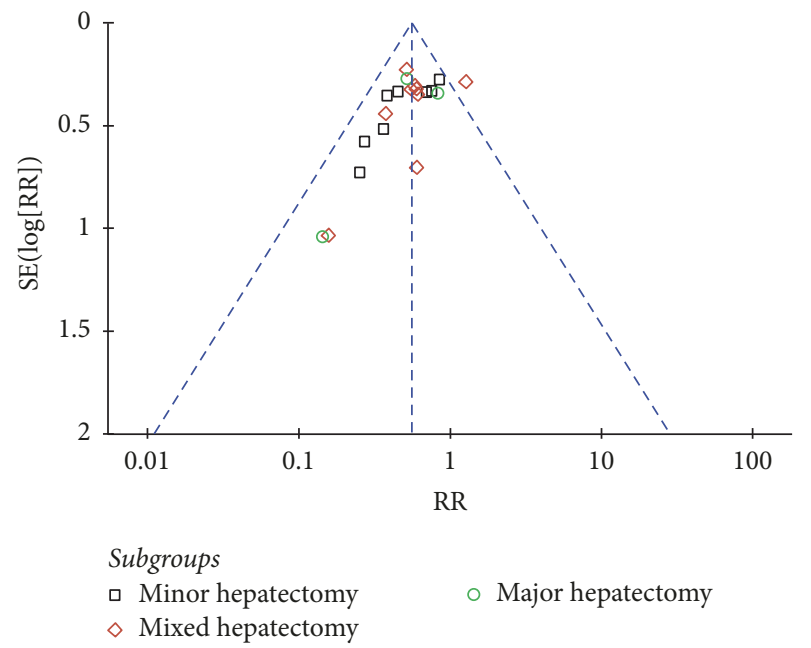

FIGURE 5: Funnel plot of the overall postoperative morbidity.

meta-analyses included all available research $[5,6,8]$ but had some limitations. Pooling of low-quality studies could undermine the strength of results, whereas selectively pooling high-quality NRCTs could strengthen the power of results [10]. Patients' characteristics and surgical extension have a major impact on the surgical outcomes of hepatectomy. Previous meta-analyses pooled studies, which did not balance the combined factors of tumor size, location, the severity of cirrhosis, and other underlying liver diseases between $\mathrm{LH}$ and $\mathrm{OH}$. These factors would have influenced the decision of surgeons and patients and further influenced the major factors of both short- and long-term outcomes. In addition, previous meta-analyses studied LH confined to minor resection. With the accumulation of surgical techniques, major resection of $\mathrm{LH}$ has become more commonly performed, but various efficacy and safety concerns for the procedure are warranted. Furthermore, since the publication of previous meta-analyses, several notable clinical observational studies have become available and some of them are from China, where HCC has the highest prevalence in the world $[8,19]$. Therefore, our comprehensive meta-analysis will contribute to a more systematic and objective evaluation of the safety and HCC treatment of LH.

Several previous studies have demonstrated that LH can be feasible and beneficial for minor resections or nonanatomical resections of peripheral HCC. This is in accordance with our study that showed minor resection of LH with similar operation time and less blood loss than $\mathrm{OH}$. However, minor hepatectomy is insufficient for large lesions or those located in posterosuperior liver segments to ensure an adequate resection margin and eliminate intrahepatic recurrence. Major hepatectomy is more frequently performed with a curative intent for multifocal or large size HCC or those with a high propensity to invade the portal vein branches [41-43]. Laparoscopic major hepatectomy is, because of the same steps and principles used in laparotomy, technically demanding. Mobilization of a heavy as well as fragile organ, excisions of bulky parenchyma, and major vascular dissection with its associated risk of major vessel injury are all considered risky under laparoscopy. As expected, the present study revealed longer operation times in laparoscopic major hepatectomy. Furthermore, unlike minor resections, the blood loss of laparoscopic major hepatectomy was not superior to its open counterpart.

Patients with HCC and concurrent cirrhosis tend to have higher incidences of postoperative complications and of greater severity. Therefore, the decreased complications in the LH group should be our most striking finding. In detail, postoperative ascites and liver failure tend to decrease in LH. Postoperative decompensation after hepatectomy 
occurs more frequently in patients with liver cirrhosis or portal hypertension, even for limited resections. The minimization of surgical incision and the subsequent preservation of abdominal wall circulation and lymphatic flow can explain fewer ascites and liver failure in LH. Moreover, a small incision limits the evacuation of ascites through the wall and decreases the risk of infection, thus facilitating wound healing. Laparoscopic surgery also decreases the manipulation of abdominal organs and exposure of bowels, which will also contribute to reduced ascites. Since refractory ascites and progressive liver insufficiency are major causes of severe postoperative morbidities, reduced severe postoperative morbidities and mortality could be expected. Major surgery was often thought to be unsuitable for those with severely impaired pulmonary function due to a higher risk of postoperative respiratory complications. Hepatectomy involving multiple systems, especially the water and electrolyte balance, is a major risk factor for medical complications. It was observed from the reviewed studies that respiratory complications were the most common medical complications, mainly pulmonary infection, followed by cardiovascular complications. Improved preservation of liver functions in LH maintains enough albumin synthesis and decreases the pleural effusion. The pain caused by large incisions, as well as the use of tension sutures and abdominal bandages after laparotomy, can make it difficult for patients to cough. Earlier postoperative ambulation in the laparoscopic group also helped to reduce respiratory complications and promote the postoperative recovery of gastrointestinal function. In accordance with other laparoscopic surgeries, LH achieved enhanced postoperative recovery. The postoperative hospitalization of LH decreases by more than two days. This can be explained by the milder surgical trauma of LH and subsequent faster bowel recovery. Less postoperative morbidities also contribute to shorter length of hospitalization.

The oncologic results of LH for HCC remain a matter of debate. Adequate surgical margins independently improve the long-term oncological outcomes. Our analysis showed that LH could achieve enough surgical margins (more than $2 \mathrm{~cm}$ ) as $\mathrm{OH}$. The 5-year OS and DFS also showed that LH was comparable to $\mathrm{OH}$. However, the results warrant prudent interpretation because of the discrepancies among the pooled studies, such as tumor size, tumor number, and status of the vascular invasion. Other biases lie in other factors including preoperative TACE and postoperative adjuvant therapies. Unfortunately, none of the three major hepatectomy studies can be included in our survival analysis. Thus, welldesigned RCTs, that balance all potential factors, preferably containing major resection are needed to confirm our results.

In the process of our research and manuscript review, two similar articles by Sotiropoulos et al. were published [44, 45], which also had limitations. Examples include pooling the low-quality studies together, failing to evaluate extension on surgical outcomes, and one paper only investigating studies conducted in Europe [45]. Besides, since these studies were published, several clinical observational studies have become available. Therefore, our comprehensive meta-analysis will contribute to a more systematic and objective evaluation of this subject.

The major limitation of this study was that all included studies are NRCTs and of retrospective design. NRCTs have potential biases that limit an unequivocal conclusion, even though we exclusively included the case-matched studies to minimize the selection biases. Another limitation is the lack of studies on laparoscopic major hepatectomy. The analysis was based on only three pooled studies. Little is known about how these results would hold for a larger sample size, which is particularly important as a fair number of patients with HCC are treated with open major hepatectomy. In addition, data from several studies are extracted using the methods reported by Hozo et al. and Tierney et al., which are not completely accurate and result in bias. Moreover, it is quite possible that surgical teams undertaking research and publishing their results are more experienced and more skillful than others. Publication bias was inevitable since one plot was outside the funnel. The bias would be overcome only with the collection of more reports.

\section{Conclusions}

This meta-analysis has highlighted that LH can be safely performed in select patients and improves surgical outcomes when compared to $\mathrm{OH}$. The data indicate that laparoscopic minor hepatectomy is acceptable with less blood loss, less postoperative morbidity, shorter hospitalization, and comparable operation times and oncological outcomes. The role of laparoscopic major hepatectomy is promising in terms of decreasing postoperative morbidity and recovery, but the technique also has drawbacks in prolonged operation time. Given the heterogeneity of the patient groups, the limitations of study design, and the small sample size, it is likely that patients have potential to benefit from LH, but further well-designed studies are needed to accurately select them.

$\begin{array}{ll}\text { Abbreviations } \\ \text { LH: } & \text { Laparoscopic hepatectomy } \\ \text { OH: } & \text { Open hepatectomy } \\ \text { HCC: } & \text { Hepatocellular carcinoma } \\ \text { NRCT: } & \text { Nonrandomized comparative study } \\ \text { RCT: } & \text { Randomized controlled trial } \\ \text { MINORS: } & \text { Methodological Index for Nonrandomized } \\ & \text { Studies } \\ \text { RR: } & \text { Risk ratio } \\ \text { WMD: } & \text { Weighted mean difference } \\ \text { SD: } & \text { Standard deviation } \\ \text { OS: } & \text { Overall survival rate } \\ \text { DFS: } & \text { Disease-free survival rate } \\ \text { HR: } & \text { Hazard ratio. }\end{array}$

\section{Conflicts of Interest}

The authors declare that they have no conflicts of interest. 


\section{Authors' Contributions}

Ke Chen designed the study; Yu Pan and Xiao-long Liu collected literature and conducted the analysis of pooled data; Hendi Maher helped to draft the manuscript; Ke Chen and Xue-yong Zheng wrote the manuscript; Xue-yong Zheng proofread and revised the manuscript. All authors have approved the version to be published.

\section{Supplementary Materials}

Table of modified MINORS score of studies with score $<12$. (Supplementary Materials)

\section{References}

[1] L. A. Torre, F. Bray, R. L. Siegel, J. Ferlay, and J. LortetTieulent, "Global cancer statistics, 2012," CA: A Cancer Journal for Clinicians, vol. 65, no. 2, pp. 87-108, 2015.

[2] J. S. Azagra, M. Goergen, E. Gilbart, and D. Jacobs, "Laparoscopic anatomical (hepatic) left lateral segmentectomy - Technical aspects," Surgical Endoscopy, vol. 10, no. 7, pp. 758-761, 1996.

[3] H. Kaneko, S. Takagi, and T. Shiba, "Laparoscopic partial hepatectomy and left lateral segmentectomy: Technique and results of a clinical series," Surgery, vol. 120, no. 3, pp. 468-475, 1996.

[4] A. Kanazawa, T. Tsukamoto, and S. Shimizu, "Impact of laparoscopic liver resection for hepatocellular carcinoma with F4-liver cirrhosis," Surgical Endoscopy, vol. 27, no. 7, pp. 2592-2597, 2013.

[5] Y.-M. Zhou, W.-Y. Shao, Y.-F. Zhao, D.-H. Xu, and B. Li, "Metaanalysis of laparoscopic versus open resection for hepatocellular carcinoma," Digestive Diseases and Sciences, vol. 56, no. 7, pp. 1937-1943, 2011.

[6] N. Li, Y.-R. Wu, B. Wu, and M.-Q. Lu, "Surgical and oncologic outcomes following laparoscopic versus open liver resection for hepatocellular carcinoma: A meta-analysis," Hepatology Research, vol. 42, no. 1, pp. 51-59, 2012.

[7] J.-J. Xiong, K. Altaf, M. A. Javed et al., "Meta-analysis of laparoscopic vs open liver resection for hepatocellular carcinoma," World Journal of Gastroenterology, vol. 18, no. 45, pp. 6657-6668, 2012.

[8] Z. Yin, X. Fan, H. Ye, D. Yin, and J. Wang, "Short- and longterm outcomes after laparoscopic and open hepatectomy for hepatocellular carcinoma: a global systematic review and metaanalysis," Annals of Surgical Oncology, vol. 20, no. 4, pp. 12031215, 2013.

[9] R. R. MacLehose, B. C. Reeves, I. M. Harvey et al., A systematic review of comparisons of effect sizes derived from randomised and non-randomised studies. Health technology assessment, vol. 34, Health Technology Assessment, Winchester, England, 2000.

[10] N. S. Abraham, C. J. Byrne, J. M. Young, and M. J. Solomon, "Meta-analysis of well-designed nonrandomized comparative studies of surgical procedures is as good as randomized controlled trials," Journal of Clinical Epidemiology, vol. 63, no. 3, pp. 238-245, 2010.

[11] K. Slim, E. Nini, D. Forestier, F. Kwiatkowski, Y. Panis, and J. Chipponi, "Methodological index for non-randomized studies (Minors): development and validation of a new instrument," ANZ Journal of Surgery, vol. 73, no. 9, pp. 712-716, 2003.
[12] S. P. Hozo, B. Djulbegovic, and I. Hozo, "Estimating the mean and variance from the median, range, and the size of a sample," BMC Medical Research Methodology, vol. 5, article 13, 2005.

[13] J. P. T. Higgins, S. G. Thompson, J. J. Deeks, and D. G. Altman, "Measuring inconsistency in meta-analyses," British Medical Journal, vol. 327, no. 7414, pp. 557-560, 2003.

[14] J. F. Tierney, L. A. Stewart, D. Ghersi, S. Burdett, and M. R. Sydes, "Practical methods for incorporating summary time-toevent data into meta-analysis," Trials, vol. 8, article 16, 2007.

[15] A. Laurent, D. Cherqui, M. Lesurtel et al., "Laparoscopic liver resection for subcapsular hepatocellular carcinoma complicating chronic liver disease," JAMA Surgery, vol. 138, no. 7, pp. 763769, 2003.

[16] E. C. H. Lai, C. N. Tang, J. P. Y. Ha, and M. K. W. Li, "Laparoscopic liver resection for hepatocellular carcinoma tenyear experience in a single center," JAMA Surgery, vol. 144, no. 2, pp. 143-147, 2009.

[17] U. Sarpel, M. M. Hefti, J. P. Wisnievsky, S. Roayaie, M. E. Schwartz, and D. M. Labow, "Outcome for patients treated with laparoscopic versus open resection of hepatocellular carcinoma: Case-matched analysis," Annals of Surgical Oncology, vol. 16, no. 6, pp. 1572-1577, 2009.

[18] L. Aldrighetti, E. Guzzetti, C. Pulitanò et al., "Case-matched analysis of totally laparoscopic versus open liver resection for HCC: Short and middle term results," Journal of Surgical Oncology, vol. 102, no. 1, pp. 82-86, 2010.

[19] B.-S. Hu, K. Chen, H.-M. Tan, X.-M. Ding, and J.-W. Tan, "Comparison of laparoscopic vs open liver lobectomy (segmentectomy) for hepatocellular carcinoma," World Journal of Gastroenterology, vol. 17, no. 42, pp. 4725-4728, 2011.

[20] M. Meguro, T. Mizuguchi, M. Kawamoto et al., "Clinical comparison of laparoscopic and open liver resection after propensity matching selection," Surgery, vol. 158, no. 3, pp. 573-587, 2015.

[21] G. Belli, C. Fantini, A. D’Agostino et al., "Laparoscopic versus open liver resection for hepatocellular carcinoma in patients with histologically proven cirrhosis: Short- and middle-term results," Surgical Endoscopy, vol. 21, no. 11, pp. 2004-2011, 2007.

[22] H. Tranchart, G. Di Giuro, P. Lainas et al., "Laparoscopic resection for hepatocellular carcinoma: A matched-pair comparative study," Surgical Endoscopy, vol. 24, no. 5, pp. 1170-1176, 2010.

[23] H. H. Kim, E. K. Park, J. S. Seoung et al., "Liver resection for hepatocellular carcinoma: Case-matched analysis of laparoscopic versus open resection," Journal of the Korean Surgical Society, vol. 80, no. 6, pp. 412-419, 2011.

[24] K. F. Lee, C. N. Chong, J. Wong, Y. S. Cheung, J. Wong, and P. Lai, "Long-term results: Of laparoscopic hepatectomy versus open hepatectomy for hepatocellular carcinoma: A casematched analysis," World Journal of Surgery, vol. 35, no. 10, pp. 2268-2274, 2011.

[25] S. Truant, A. F. Bouras, M. Hebbar et al., "Laparoscopic resection vs. open liver resection for peripheral hepatocellular carcinoma in patients with chronic liver disease: A casematched study," Surgical Endoscopy, vol. 25, no. 11, pp. 36683677, 2011.

[26] K. S. Ahn, K. J. Kang, Y. H. Kim, T.-S. Kim, and T. J. Lim, "A propensity score-matched case-control comparative study of laparoscopic and open liver resection for hepatocellular carcinoma," Journal of Laparoendoscopic \& Advanced Surgical Techniques, vol. 24, no. 12, pp. 872-877, 2014.

[27] H. Kim, K.-S. Suh, K.-W. Lee et al., "Long-term outcome of laparoscopic versus open liver resection for hepatocellular 
carcinoma: A case-controlled study with propensity score matching," Surgical Endoscopy, vol. 28, no. 3, pp. 950-960, 2014.

[28] R. Memeo, N. De’Angelis, P. Compagnon et al., "Laparoscopic vs. open liver resection for hepatocellular carcinoma of cirrhotic liver: A case-control study," World Journal of Surgery, vol. 38, no. 11, pp. 2919-2926, 2014.

[29] H.-S. Han, A. Shehta, S. Ahn, Y.-S. Yoon, J. Y. Cho, and Y. Choi, "Laparoscopic versus open liver resection for hepatocellular carcinoma: Case-matched study with propensity score matching," Journal of Hepatology, vol. 63, no. 3, article no. 5641, pp. 643-650, 2015.

[30] B. Lau, C. Franken, D. Lee, K. Putchakayla, and L. A. DiFronzo, "Short-term outcomes of laparoscopic versus open formal anatomical hepatectomy: A case matched control study," The American Surgeon, vol. 81, no. 10, pp. 1097-1100, 2015.

[31] J. J. Lee, J. B. Conneely, and R. L. Smoot, "Laparoscopic versus open liver resection for hepatocellular carcinoma at a NorthAmerican Centre: a 2-to-1 matched pair analysis," HPB: The Official Journal of the International Hepato Pancreato Biliary Association, vol. 17, no. 4, pp. 304-310, 2015.

[32] L. Luo, H. Zou, Y. Yao, and X. Huang, "Laparoscopic versus open hepatectomy forhepatocellular carcinoma: Short- and longterm outcomes comparison," International Journal of Clinical and Experimental Medicine, vol. 8, no. 10, pp. 18772-18778, 2015.

[33] T. Takahara, G. Wakabayashi, T. Beppu et al., "Long-term and perioperative outcomes of laparoscopic versus open liver resection for hepatocellular carcinoma with propensity score matching: A multi-institutional Japanese study," Journal of Hepato-Biliary-Pancreatic Sciences, vol. 22, no. 10, pp. 721-727, 2015.

[34] S.-Y. Yoon, K.-H. Kim, D.-H. Jung, A. Yu, and S.-G. Lee, "Oncological and surgical results of laparoscopic versus open liver resection for HCC less than $5 \mathrm{~cm}$ : case-matched analysis," Surgical Endoscopy, vol. 29, no. 9, pp. 2628-2634, 2015.

[35] T. T. Cheung, W. C. Dai, S. H. Y. Tsang et al., "Pure laparoscopic hepatectomy versus open hepatectomy for hepatocellular carcinoma in 110 patients with liver cirrhosis: A propensity analysis at a single center," Annals of Surgery, vol. 264, no. 4, pp. 612-620, 2016.

[36] X. Jiang, L. Liu, Q. Zhang et al., "Laparoscopic versus open hepatectomy for hepatocellular carcinoma: long-term outcomes," Journal of BUON : official journal of the Balkan Union of Oncology, vol. 21, no. 1, pp. 135-141, 2016.

[37] S. Komatsu, R. Brustia, C. Goumard, F. Perdigao, O. Soubrane, and O. Scatton, "Laparoscopic versus open major hepatectomy for hepatocellular carcinoma: a matched pair analysis," Surgical Endoscopy, vol. 30, no. 5, pp. 1965-1974, 2016.

[38] C. Sposito, C. Battiston, A. Facciorusso et al., "Propensity score analysis of outcomes following laparoscopic or open liver resection for hepatocellular carcinoma," British Journal of Surgery, vol. 103, no. 7, pp. 871-880, 2016.

[39] H.-W. Xu, F. Liu, H.-Y. Li, Y.-G. Wei, and B. Li, "Outcomes following laparoscopic versus open major hepatectomy for hepatocellular carcinoma in patients with cirrhosis: a propensity score-matched analysis," Surgical Endoscopy, pp. 1-8, 2017.

[40] Y.-I. Yoon, K.-H. Kim, S.-H. Kang et al., "Pure Laparoscopic Versus Open Right Hepatectomy for Hepatocellular Carcinoma in Patients with Cirrhosis," Annals of Surgery, vol. 265, no. 5, pp. 856-863, 2017.

[41] D. Dahiya, T.-J. Wu, C.-F. Lee, K.-M. Chan, W.-C. Lee, and M.-F. Chen, "Minor versus major hepatic resection for small hepatocellular carcinoma (HCC) in cirrhotic patients: A 20year experience," Surgery, vol. 147, no. 5, pp. 676-685, 2010.

[42] K. Hasegawa, N. Kokudo, H. Imamura et al., "Prognostic impact of anatomic resection for hepatocellular carcinoma," Annals of Surgery, vol. 242, no. 2, pp. 252-259, 2005.

[43] B. H.-H. Lang, R. T.-P. Poon, S.-T. Fan, and J. Wong, "Perioperative and long-term outcome of major hepatic resection for small solitary hepatocellular carcinoma in patients with cirrhosis," JAMA Surgery, vol. 138, no. 11, pp. 1207-1213, 2003.

[44] G. C. Sotiropoulos, A. Prodromidou, I. D. Kostakis, and N. Machairas, "Meta-analysis of laparoscopic vs open liver resection for hepatocellular carcinoma," Updates in Surgery, vol. 69, no. 3, pp. 291-311, 2017.

[45] G. C. Sotiropoulos, A. Prodromidou, and N. Machairas, "Machairas N: Meta-analysis of laparoscopic vs open liver resection for hepatocellular carcinoma: The European experience," Journal of BUON : Official Journal of the Balkan Union of Oncology, vol. 22, no. 5, pp. 1160-1171, 2017. 


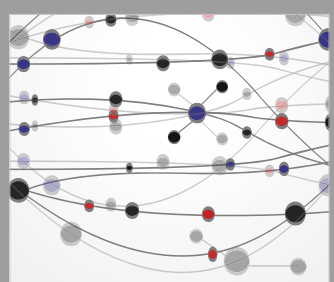

The Scientific World Journal
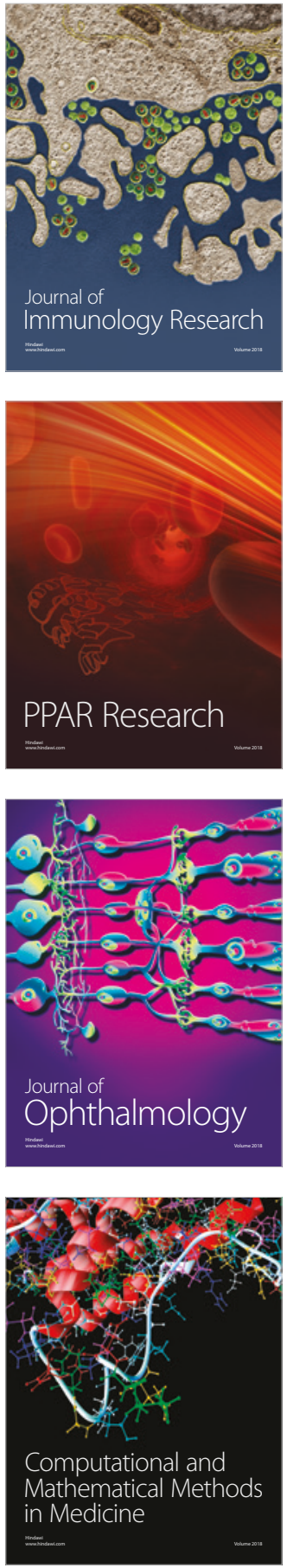

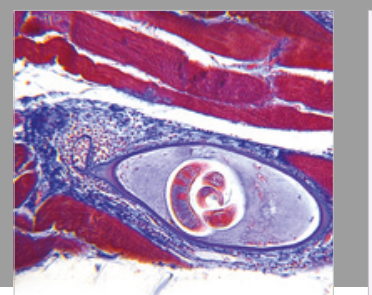

Gastroenterology Research and Practice

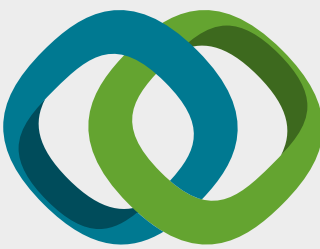

\section{Hindawi}

Submit your manuscripts at

www.hindawi.com
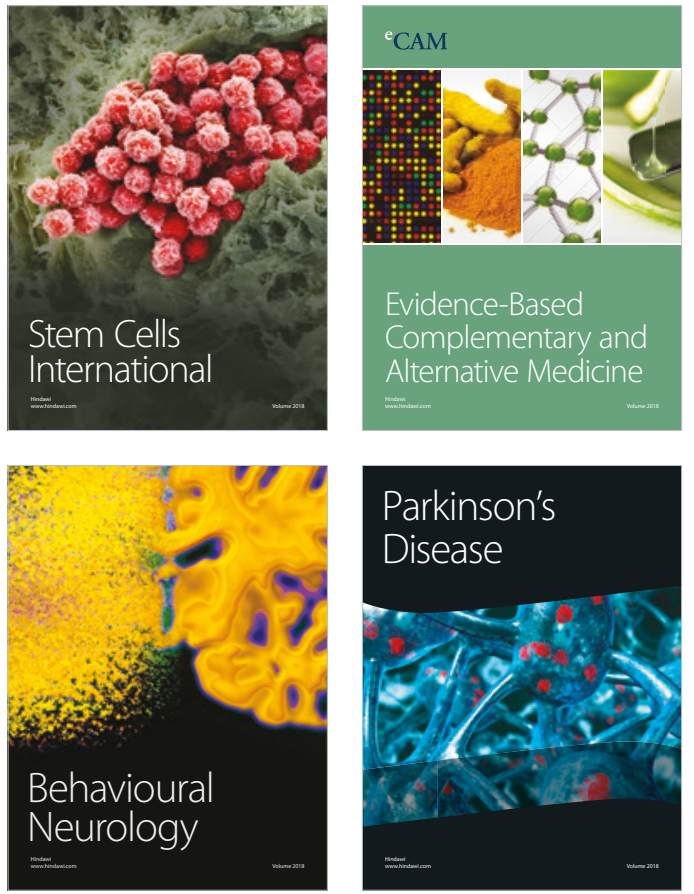

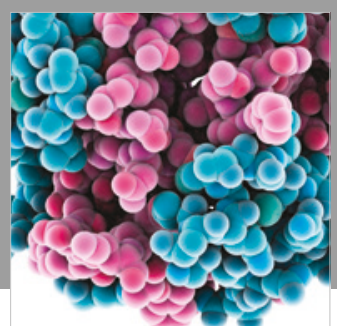

ournal of

Diabetes Research

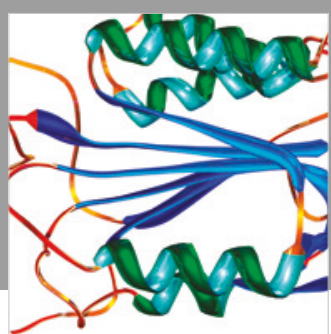

Disease Markers
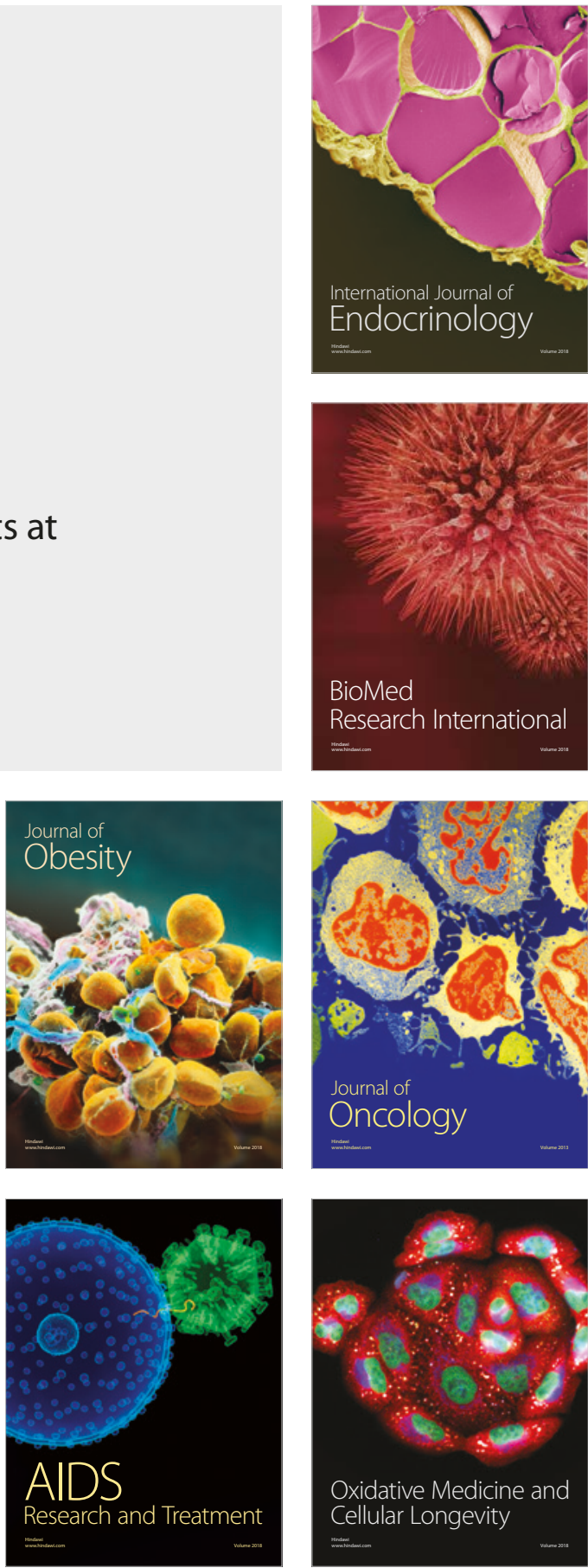